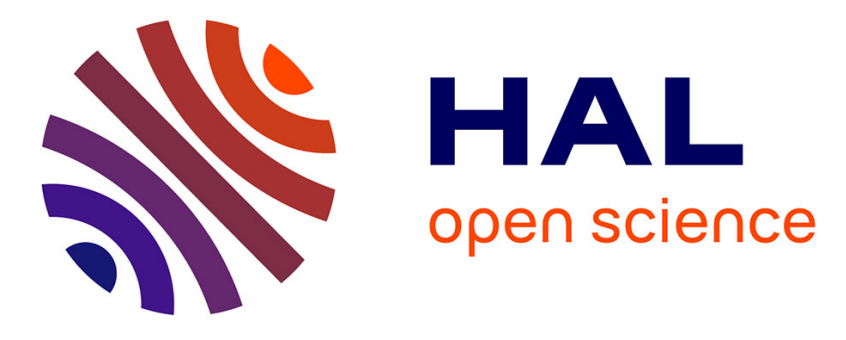

\title{
Online deconvolution for pushbroom hyperspectral imaging systems
}

Yingying Song, El-Hadi Djermoune, Jie Chen, Cédric Richard, David Brie

\section{To cite this version:}

Yingying Song, El-Hadi Djermoune, Jie Chen, Cédric Richard, David Brie. Online deconvolution for pushbroom hyperspectral imaging systems. 7th IEEE International Workshop on Computational Advances in Multi-Sensor Adaptive Processing, CAMSAP 2017, IEEE, Dec 2017, Curaçao, Netherlands Antilles. hal-01493901v2

\section{HAL Id: hal-01493901 \\ https://hal.science/hal-01493901v2}

Submitted on 5 Feb 2018

HAL is a multi-disciplinary open access archive for the deposit and dissemination of scientific research documents, whether they are published or not. The documents may come from teaching and research institutions in France or abroad, or from public or private research centers.
L'archive ouverte pluridisciplinaire HAL, est destinée au dépôt et à la diffusion de documents scientifiques de niveau recherche, publiés ou non, émanant des établissements d'enseignement et de recherche français ou étrangers, des laboratoires publics ou privés. 


\title{
Online deconvolution for pushbroom hyperspectral imaging systems
}

\author{
Yingying Song*, El-Hadi Djermoune*, Jie Chen ${ }^{\dagger}$, Cédric Richard ${ }^{\ddagger}$ and David Brie* \\ *CRAN, Université de Lorraine, CNRS, Vandœuvre-lès-Nancy, France \\ Email: firstname.lastname@univ-lorraine.fr \\ $\dagger$ Northwestern Polytechnical University, Xi’an, China \\ Email: dr.jie.chen@ieee.org \\ ‡Université Côte d'Azur, CNRS, OCA, Nice, France \\ Email: cedric.richard@unice.fr
}

\begin{abstract}
This paper introduces a framework based on the LMS algorithm for sequential deconvolution of hyperspectral images acquired by industrial pushbroom imaging systems. Considering a sequential model of image blurring phenomenon, we derive a sliding-block zero-attracting LMS algorithm with spectral regularization. The role of each hyper-parameter is discussed. The performance of the algorithm is evaluated using real hyperspectral data.
\end{abstract}

\section{INTRODUCTION}

Hyperspectral imaging has received considerable attention in the last decade as it combines the power of digital imaging and spectroscopy. Every pixel in a hyperspectral image provides local spectral information about a scene of interest across a large number of contiguous bands. This information can be used to characterize objects with great precision and detail in a number of areas, including agricultural monitoring, industrial inspection, and defense. The core characteristics of hyperspectral images raise new data processing issues ranging from image restoration to pattern recognition [1], [2]. Several sensing techniques have been developed for hyperspectral imaging. They can be categorized into four main groups [3], [4]: whiskbroom (point scan), pushbroom (line scan), tunable filter (wavelength scan), and snapshot.

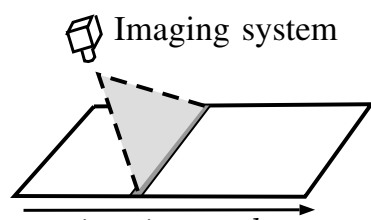

Time instant $k$

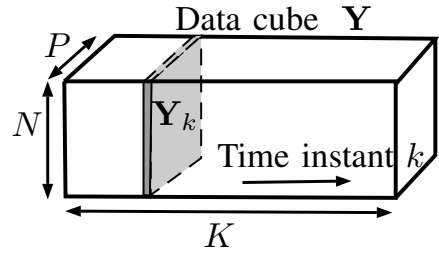

Fig. 1: Data acquisition by a pushbroom imaging system

Figure 1 depicts schematically how a hyperspectral image is captured by a pushbroom imager, and how the spatialspectral arrays are stacked within a hyperspectral image datacube. With the pushbroom technique, pixel spectra are sensed line-by-line at each time instant. The scene is typically scanned by moving the imager or its field of view across the scene. Pushbroom systems are used in many areas such as

This work has been supported by the FUI AAP 2015 Trispirabois Project, the Conseil Régional de Lorraine and the GDR ISIS. food safety [5], [6], georeferencing [7] and material sorting [8], [9]. This paper is a first step towards the development of advanced online hyperspectral image processing methods which are required in industrial processes aiming at controlling and sorting input materials right after each line scanning. For example, this is the case for paper plants and chipboard plants that use recycled materials in the manufacturing process. The aim of this paper is to address the fast online (sequential) deconvolution of hyperspectral images provided by pushbroom imaging systems ${ }^{1}$ to improve image resolution. Multichannel image restoration was carried out with Wiener methods in [10], [11]. Other strategies such as those in [12]-[15] were also introduced, but only in an offline setting. Let us mention the works of Jemec et al. [16], [17] which specifically address the calibration and (offline) deconvolution of pushbroom hyperspectral images.

Consider a hyperspectral image $\mathbf{Y} \in \mathbb{R}^{N \times P \times K}$ collected by a pushbroom hyperspectral imaging system, where $N, P$, and $K$ denote the number of spatial, spectral and time measurements, respectively. The samples to be imaged are carried by a conveyor moving at constant speed. The hyperspectral image is then obtained slice by slice, each is denoted by $\mathbf{Y}_{k} \in \mathbb{R}^{N \times P}$, $k=1, \ldots, K$. The size of $\mathbf{Y}$ increases with $k$ which can possibly grow to infinity. The acquisition parameters are the spectral sampling $\Delta_{\lambda}$ (controlled by the spectral binning), the spatial sampling $\Delta_{s}$ and integration time $T$. We consider situations where the spectral response is smooth enough to choose a spectral sampling $\Delta_{\lambda}$ greater than the support of the spectral blurring, a spatial sampling $\Delta_{s}$ smaller than the support of the point spread function and a short integration time (for fast scanning). This results in a degradation model involving only spatial blurring (possibly depending on the wavelength) of the hyperspectral image and a low signal to noise ratio (SNR). The main contribution of this work is to introduce an LMS framework for sequential deconvolution of hyperspectral images. While our algorithm operates in the spirit of LMS-based algorithms used, for instance, for adaptive system identification [18]-[20] and super-resolution restoration of image sequences [21], [22], sequential image deconvolution of hyperspectral images based on the LMS has never been reported in the literature. Accounting for the specificity of

\footnotetext{
${ }^{1}$ It is worth to be noted that the proposed sequential deconvolution algorithm can straightforwardly be extended to whiskbroom systems.
} 
the degradation process, we propose a sliding-block LMS algorithm that allows to sequentially restore the hyperspectral image of interest with a delay $Q$. We also introduce a zero attracting regularization term promoting the restoration of objects on a background which should be zero-valued.

\section{BLURRING AND CAUSALITY ISSUES}

We shall now discuss issues related to the causality of convolution kernel and associated estimates. Following [14], hyperspectral image blurring can be seen as $P$ simultaneous spatial convolutions. For each wavelength $\lambda_{p}$, the blurred spatial image $\mathbf{Y}^{p} \in \mathbb{R}^{N \times K}$ is given by the $2 \mathrm{D}$ convolution:

$$
\mathbf{Y}^{p}=\overline{\mathbf{H}}^{p} * \mathbf{X}^{p}+\mathbf{Z}^{p}
$$

where $*$ is the $2 \mathrm{D}$ convolution operator, $\mathbf{X}^{p} \in \mathbb{R}^{N \times K}$ is the image to restore, $\overline{\mathbf{H}}^{p} \in \mathbb{R}^{M \times L}$ is a convolution kernel (filter), and $\mathbf{Z}^{p}$ is a noise supposed to be additive and i.i.d. We first derive a sequential causal formulation of model (1). Without loss of generality, we shall focus on the sequential model for $2 \mathrm{D}$ images, by omitting the dependence with respect to $p$. The image $\mathbf{Y}$, collected in an online way, can be represented as a sequence of vectors $\mathbf{y}_{k}:=\left[y_{1, k}, \ldots, y_{N, k}\right]^{\top}, k=1, \ldots, K$, where ${ }^{\top}$ denotes the transpose of a matrix. We shall use the same notation for $\mathbf{X}$. We assume a finite length blurring kernel of size $L$ along the time dimension, centered around 0 which means that past and future values of $\mathbf{x}_{k}$ contribute to the observation $\mathbf{y}_{k}$. In order to make the blurring kernel causal $^{2}$, it has to be shifted by $(L-1) / 2$, which means that the observations needs to be delayed by $(L-1) / 2$ samples, that is, $\tilde{\mathbf{y}}_{k}=\mathbf{y}_{k-(L-1) / 2}$. Writing $\overline{\mathbf{H}}=\left[\mathbf{h}_{L}, \ldots, \mathbf{h}_{1}\right]$ with $\mathbf{h}_{\ell}=\left[h_{M, \ell}, \ldots, h_{1, \ell}\right]^{\top}$, model (1) can be expressed as:

$$
\tilde{\mathbf{y}}_{k}=\mathbf{y}_{k-(L-1) / 2}=\sum_{\ell=1}^{L} \mathbf{H}_{\ell} \mathbf{x}_{k-\ell+1}+\mathbf{z}_{k}
$$

where $\mathbf{z}_{k}$ is a zero-mean measurement noise, statistically independent of the other signals. $\mathbf{H}_{\ell}$ is the $N \times N$ Toeplitz matrix with first column and first row given by $\left[h_{1, \ell}, \ldots, h_{M, \ell}, 0, \ldots, 0\right]$ and $\left[h_{1, \ell}, 0, \ldots, 0\right]$, respectively. Relation (2) introduces a delay in both time dimension and spatial dimension because the filter is made causal along these two dimensions. Another consequence of causality issues concerns the estimation process of $\mathbf{x}_{k}$. First, $\mathbf{x}_{k}$ is involved in past and future observations $\left(\mathbf{y}_{k-(L-1) / 2}, \ldots, \mathbf{y}_{k}, \ldots, \mathbf{y}_{k+(L-1) / 2}\right)$. Secondly, let us consider the problem of estimating only $\mathbf{x}_{k}$ from the dataset $\tilde{\mathbf{y}} \triangleq \operatorname{col}\left\{\tilde{\mathbf{y}}_{k}\right\}_{k=1}^{K}$ where $\operatorname{col}\{\cdot\}$ stacks its vector arguments on top of each other. The least squares criterion can be written as $\|\tilde{\mathbf{y}}-\mathbf{F} \mathbf{x}\|^{2}$ where $\mathbf{x}$ is built similarly to $\tilde{\mathbf{y}}$ and $\mathbf{F}$ is a Toeplitz-block-Toeplitz matrix of proper dimensions. To make the dependence of the criterion on $\mathbf{x}_{k}$ explicit, we introduce the following partitions $\mathbf{x}=\left[\mathbf{x}_{1: k-1}^{\top}, \mathbf{x}_{k}^{\top}, \mathbf{x}_{k+1: K}^{\top}\right]^{\top}$ and $\mathbf{F}=\left[\mathbf{F}_{1: k-1}, \mathbf{F}_{k}, \mathbf{F}_{k+1: K}\right]$ where $\mathbf{x}_{i: j} \triangleq \operatorname{col}\left\{\mathbf{x}_{k}\right\}_{k=i}^{j}$ and $\mathbf{F}_{i: j}$ is the matrix formed using the columns $(i-1) N+1$ through $j N$. This results in $\|\tilde{\mathbf{y}}-\mathbf{F} \mathbf{x}\|^{2}=\left\|\tilde{\mathbf{y}}-\mathbf{F}_{1: k-1} \mathbf{x}_{1: k-1}-\mathbf{F}_{k+1: K} \mathbf{x}_{k+1: K}-\mathbf{F}_{k} \mathbf{x}_{k}\right\|^{2}$. It is now clear that optimally estimating $\mathbf{x}_{k}$ requires all the past estimations $\hat{\mathbf{x}}_{1: k-1}$ and future estimations $\hat{\mathbf{x}}_{k+1: K}$, which precludes the estimation of $\mathbf{x}_{k}$ in a sequential manner. To address these issues, we recommend to produce the estimates $\hat{\mathbf{x}}_{k}$

\footnotetext{
${ }^{2}$ For simplicity, $L$ is assumed to be odd.
}

with a delay $Q$. This means that we shall estimate $\mathbf{x}_{k-Q+1}$ given $\left(\mathbf{y}_{k+(L-1) / 2-Q+1}, \ldots, \mathbf{y}_{k+(L-1) / 2}\right)$, coarse posterior estimates $\left(\hat{\mathbf{x}}_{k}, \hat{\mathbf{x}}_{k-1}, \ldots, \hat{\mathbf{x}}_{k-Q+2}\right)$ refined as $k$ increases, and past estimates $\left(\hat{\mathbf{x}}_{k-Q}, \hat{\mathbf{x}}_{k-Q-1}, \ldots, \hat{\mathbf{x}}_{k-Q-L+2}\right)$ which are no more updated. This is the key idea of the sliding-block LMS deconvolution algorithm developed in the next section.

\section{ONLINE IMAGE DECONVOLUTION}

We shall first address the adaptive deconvolution problem in the case of 2D images. Then the proposed algorithm will be extended to hyperspectral images. Consider the problem of estimating $\mathbf{x}_{k-Q+1}$ in a sequential manner based on observations $\left(\mathbf{y}_{k+(L-1) / 2-Q+1}, \ldots, \mathbf{y}_{k+(L-1) / 2}\right)$ or, equivalently, on the delayed ones $\left(\tilde{\mathbf{y}}_{k-Q+1}, \ldots, \tilde{\mathbf{y}}_{k}\right)$. In what follows, to simplify notations, $\mathbf{y}_{k}$ refers to the delayed observation $\tilde{\mathbf{y}}_{k}$. To account for their dependencies on $\mathbf{x}_{k}, \ldots, \mathbf{x}_{k-Q-L+2}$, we consider the following criterion where, for clarity, the variables are partitioned in two sets (those to be updated and those fixed):

$$
\begin{aligned}
& \mathcal{J}(\overbrace{\mathbf{x}_{k}, \ldots, \mathbf{x}_{k-Q+1}}^{\text {updated }}, \overbrace{\mathbf{x}_{k-Q}, \ldots, \mathbf{x}_{k-Q-L+2}}^{\text {fixed }})= \\
& \sum_{q=1}^{Q} \mathbb{E}\left\|\mathbf{y}_{k-q+1}-\sum_{\ell=1}^{L} \mathbf{H}_{\ell} \mathbf{x}_{k-q-\ell+2}\right\|^{2}+\eta_{z}\left\|\mathbf{x}_{k-q+1}\right\|_{1}
\end{aligned}
$$

where $\|\cdot\|_{1}=\sum_{n=1}^{N}\left|\{\cdot\}_{n}\right|$ denotes the $\ell_{1}$-norm, and $\{\cdot\}_{n}$ stands for the $n$-th entry of a vector. The zero-attracting regularizer $\left\|\mathbf{x}_{k-q+1}\right\|_{1}$, whose strength is controlled by $\eta_{z} \geq 0$, promotes the removal of the conveyor background, and is motivated by the targeted application, namely, the inspection of objects put on the conveyor belt. At a given wavelength, the response of the conveyor after background removal is close to zero while that of the objects is not.

\section{A. Sliding-block zero-attracting LMS (SBZA-LMS)}

Consider vectorized data: $\mathbf{x}_{k}^{\prime} \triangleq \operatorname{col}\left\{\mathbf{x}_{k-q+1}\right\}_{q=1}^{Q+L-1}, \mathbf{y}_{k}^{\prime} \triangleq$ $\operatorname{col}\left\{\mathbf{y}_{k-q+1}\right\}_{q=1}^{Q+L-1}$. A subgradient of (3) is given by:

$$
\nabla \mathcal{J}\left(\mathbf{x}_{k}^{\prime}\right) \triangleq \operatorname{col}\left\{\frac{\partial \mathcal{J}}{\partial \mathbf{x}_{k}}, \ldots, \frac{\partial \mathcal{J}}{\partial \mathbf{x}_{k-Q+1}}, \mathbf{0}_{(L-1) N \times 1}\right\}
$$

where $\mathbf{0}_{I \times J}$ denotes the $I \times J$ zero matrix. Note that it is necessary to include the variables $\hat{\mathbf{x}}_{k-Q}, \hat{\mathbf{x}}_{k-Q-1}, \ldots, \hat{\mathbf{x}}_{k-Q-L+2}$ in $\mathbf{x}_{k}^{\prime}$ because $\mathbf{x}_{k-Q+1}$ depends on them. But as they are fixed, the subgradient w.r.t. these variables is equal to zero. Approximating the subgradient in (4) by its instantaneous value yields:

$$
\nabla \mathcal{J}\left(\mathbf{x}_{k}^{\prime}\right)=-2 \boldsymbol{\Phi}\left(\mathbf{y}_{k}^{\prime}-\mathbf{G x}_{k}^{\prime}\right)+\eta_{z} \operatorname{sign}\left(\mathbf{x}_{k}^{\prime}\right)
$$

where $\boldsymbol{\Phi}$ and $\mathbf{G}$ are matrices of size $(Q+L-1) N \times(Q+$ $L-1) N$. Matrix $\boldsymbol{\Phi}$ is given by:

$$
\boldsymbol{\Phi} \triangleq\left[\begin{array}{ccc|c}
\mathbf{H}_{1}^{\top} & & \mathbf{0} & \\
\vdots & \ddots & & \mathbf{0}_{Q N \times(L-1) N} \\
\mathbf{H}_{Q}^{\top} & \cdots & \mathbf{H}_{1}^{\top} & \\
\hline & \mathbf{0}_{(L-1) N \times Q N} & & \mathbf{0}_{(L-1) N \times(L-1) N}
\end{array}\right],
$$

with $\mathbf{H}_{\ell}=\mathbf{0}_{N \times N}$ for $\ell>L$, and $\mathbf{G}$ a Toeplitz-block-Toeplitz matrix whose first block column is $\left[\mathbf{H}_{1}, \mathbf{0}_{N \times N}, \ldots, \mathbf{0}_{N \times N}\right]$ 
and first block row is $\left[\mathbf{H}_{1}, \ldots, \mathbf{H}_{L}, \mathbf{0}_{N \times N}, \ldots, \mathbf{0}_{N \times N}\right]$. The sign function is defined as $\operatorname{sign}(x)=0$ for $x=0$, and $\operatorname{sign}(x)=x /|x|$ otherwise. Finally, the SBZA-LMS algorithm for image deconvolution is given by:

$$
\begin{aligned}
\hat{\mathbf{x}}_{k+1}^{\prime} & =\boldsymbol{\Omega} \hat{\mathbf{x}}_{k}^{\prime}-\frac{\mu}{2} \nabla \mathcal{J}\left(\hat{\mathbf{x}}_{k}^{\prime}\right) \\
& =\boldsymbol{\Omega} \hat{\mathbf{x}}_{k}^{\prime}+\mu \boldsymbol{\Phi}\left(\mathbf{y}_{k}^{\prime}-\mathbf{G} \hat{\mathbf{x}}_{k}^{\prime}\right)-\rho_{z} \operatorname{sign}\left(\hat{\mathbf{x}}_{k}^{\prime}\right)
\end{aligned}
$$

where $\mu$ is a step size parameter that controls the balance between convergence rate and algorithm stability. The matrix $\Omega$ is given by

$$
\boldsymbol{\Omega} \triangleq\left[\begin{array}{llll}
\mathbf{I}_{(Q-1) N} & \mathbf{0} & \mathbf{0} & \mathbf{0}_{(Q-1) N \times N} \\
\mathbf{0} & \mathbf{I}_{N} & \mathbf{0} & \mathbf{0}_{N \times N} \\
\hline \mathbf{0} & \mathbf{I}_{N} & \mathbf{0} & \mathbf{0}_{N \times N} \\
\mathbf{0} & \mathbf{0} & \mathbf{I}_{(L-2) N} & \mathbf{0}_{(L-2) N \times N}
\end{array}\right],
$$

where $\mathbf{I}_{J}$ denotes the $J \times J$ identity matrix. The upper part of matrix $\Omega$ corresponds to the set of updated variables and the lower part corresponds to the set of fixed variables. The parameter $\rho_{z}=\mu \eta_{z} / 2$. The final result $\hat{\mathbf{x}}_{k-Q+2}$ is obtained by selecting the $Q$-th block of vector $\hat{\mathbf{x}}_{k+1}^{\prime}$, that is,

$$
\hat{\mathbf{x}}_{k-Q+2}=\mathbf{S} \hat{\mathbf{x}}_{k+1}^{\prime}
$$

where $\mathbf{S} \triangleq\left[\mathbf{0}_{N \times(Q-1) N}, \mathbf{I}_{N}, \mathbf{0}_{N \times(L-1) N}\right]$. It is worth to mention that the proposed algorithm is different from the standard block-LMS algorithm for which the output $\mathbf{x}_{k}$ is updated once for every block of size $Q$. On the contrary, in the proposed algorithm, to account for the causality issues discussed in section II, $\mathbf{x}_{k}$ is updated $Q$ times. When $Q=1$, algorithm (6) reduces to the Zero-Attracting LMS (ZA-LMS) algorithm proposed in [19] for sparse system identification.

\section{B. Online hyperspectral image deconvolution}

Consider now the problem of 3D hyperspectral image deconvolution, which aims at restoring sequentially spatialspectral arrays $\mathbf{X}_{k} \in \mathbb{R}^{N \times P}$. In an equivalent way, we shall consider vectorized data

$$
\mathbf{x}_{k}^{\prime} \triangleq \operatorname{col}\left\{\mathbf{x}_{k}^{\prime p}\right\}_{p=1}^{P}, \quad \mathbf{y}_{k}^{\prime} \triangleq \operatorname{col}\left\{\mathbf{y}_{k}^{\prime p}\right\}_{p=1}^{P}
$$

where superscript $p$ refers to the spectral band. Adding a spectral regularization term to promote spectral smoothness of the image leads to the criterion:

$$
\mathcal{C}\left(\mathbf{x}_{k}^{\prime}\right)=\sum_{p=1}^{P} \mathcal{J}\left(\mathbf{x}_{k}^{\prime p}\right)+\eta_{\lambda}\left\|\boldsymbol{\Lambda}_{\lambda} \mathbf{x}_{k}^{\prime}\right\|^{2}
$$

where $\boldsymbol{\Lambda}_{\lambda} \triangleq\left(\operatorname{diag}\left(c_{1} \ldots, c_{P-1}\right) \mathbf{D}_{P}\right) \otimes \mathbf{I}_{(Q+L-1) N}$. The operator $\otimes$ stands for the Kronecker product and $\mathbf{D}_{P}$ is the Toeplitz matrix of size $(P-1) \times P$ with first column $[1,0, \ldots, 0]$ and first row $[1,-1,0, \ldots, 0]$. The matrix $\boldsymbol{\Lambda}_{\lambda}$ is a first-order filtering operator along the spectral dimension weighted by the coefficients $\left\{c_{p}\right\}_{p=1}^{P-1}$. The parameter $\eta_{\lambda}$ controls the strength of the spectral smoothness penalty term. Finally, the SBZALMS algorithm for hyperspectral image deconvolution can be expressed as:

$$
\begin{aligned}
\hat{\mathbf{x}}_{k+1}^{\prime} & =\boldsymbol{\Gamma} \hat{\mathbf{x}}_{k}^{\prime}+\mu \boldsymbol{\Psi}\left(\mathbf{y}_{k}^{\prime}-\boldsymbol{\Upsilon} \hat{\mathbf{x}}_{k}^{\prime}\right)-\rho_{z} \operatorname{sign}\left(\hat{\mathbf{x}}_{k}^{\prime}\right) \\
& -\mu \eta_{\lambda} \boldsymbol{\Lambda}_{\lambda}^{\top} \boldsymbol{\Lambda}_{\lambda} \hat{\mathbf{x}}_{k}^{\prime}
\end{aligned}
$$

with $\boldsymbol{\Gamma} \triangleq \mathbf{I}_{P} \otimes \boldsymbol{\Omega}, \boldsymbol{\Psi} \triangleq \operatorname{blkdiag}\left\{\boldsymbol{\Phi}^{p}\right\}_{p=1}^{P}$ a block-diagonal matrix and $\boldsymbol{\Upsilon} \triangleq \operatorname{blkdiag}\left\{\mathbf{G}^{p}\right\}_{p=1}^{P}$.

\section{Comments}

The statistical analysis of the SBZA-LMS algorithm performed in [23] provides an accurate model for its steady-state and non-stationary behavior. Based on this model, we were able to derive stability conditions for the algorithm, and to analyze the influence of the hyperparameters $\left(Q, \mu, \rho_{z}, \eta_{\lambda}\right)$. We also evaluated its computational complexity. We give here the main conclusions: ( $i$ ) following the lines of [24], it can be proved that the stability of the SBZA-LMS is essentially controlled by the stability of the SB-LMS; (ii) the best tradeoff between accuracy and computational cost is obtained for $Q=L$; (iii) the SB-LMS $\left(\rho_{z}=0\right)$ can be interpreted as a delayed Tikhonov-like algorithm (with regularization along time and spectral dimensions) where the time regularization parameter is proportional to the inverse of $\mu$. This equivalence can be formally proved for the denoising problem (i.e. identity convolution kernel); (iv) the ZA term plays a key role to recover the background at low noise levels. It induces an asymmetric transient behavior: as compared to the SB-LMS, the convergence of the SBZA-LMS is faster when starting from a non-zero value toward a zero value and slower when starting from a zero value toward a non-zero value.

\section{EXPERIMENTAL RESULTS}

To assess the influence of the hyperparameters $Q, \mu$ and $\rho_{z}$, numerical simulations are conducted on a $2 \mathrm{D}$ image. The original image is blurred by a Gaussian kernel of size $15 \times 15$ with full width at half-maximum set to 7 pixels. A Gaussian noise is added to reach a $10 \mathrm{~dB}$ SNR. This blurred and noisy image is shown in Figure 2(a). Figures 2(b) to 2(d) show the results corresponding to the ZA-LMS algorithm obtained by setting $Q=1, \mu=0.06$ and $\rho_{z}=0.02$, the SB-LMS algorithm (sliding block LMS without zero-attracting term) with hyperparameters $Q=15, \mu=0.006$ and the SBZA-LMS with hyperparameters $Q=15, \mu=0.006$ and $\rho_{z}=0.05$. The image restored with ZA-LMS in Figure 2(b) exhibits lower noise level than the original image but deblurring is limited. Better results were obtained when the block size increases as shown in Figure 2(c). The image restored by SBZA-LMS has a better resolution and a lower noise level as illustrated in Fig. 2(d). As mentioned in section III-C, the SB-LMS and SBZA-LMS induce a time (and no spatial) regularization resulting in horizontal structures visible on the restored images. This effect can be attenuated by introducing a spatial regularization (see [23] for details). Figures 2(e) and 2(f) show the evolution of the mean squared error (MSE) as a function of $\mu$ and $\eta_{z}$ respectively for different values of the SNR. For each value of SNR, the MSE has a minimum value (red point) corresponding to the best tradeoff between bias and variance: the optimal value of $\mu$ decreases as the noise level increases and the optimal value of $\rho_{z}$ increases as the noise level increases which conforms with intuition. Results obtained (but not presented here) for the spectral regularization parameter $\rho_{\lambda}$ are similar to that of $\rho_{z}$.

The second experiment aims at illustrating the performance of the SBZA-LMS algorithm on a real blurred hyperspectral image of size $121 \times 171 \times 16$ with wavelengths varying from $501.1 \mathrm{~nm}$ to $868.6 \mathrm{~nm}$ with an increment of $24.5 \mathrm{~nm}$. The spectral response of the conveyor (background) was estimated from data in an area of size $120 \times 120$. It was then subtracted 


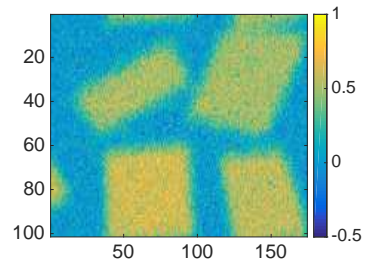

(a) Blurred noisy image

(c) Restored by SB-LMS

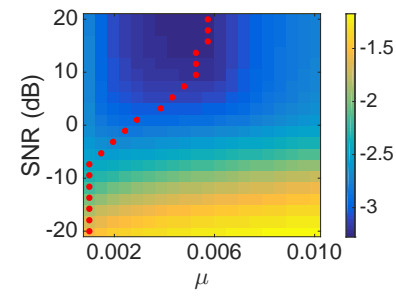

(e) MSE as a function of $\mu$

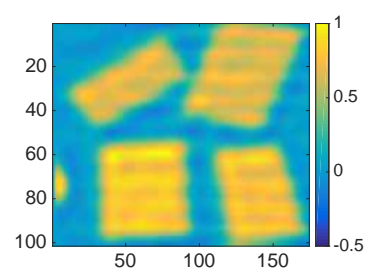
$\left(\rho_{z}=0, Q=15\right)$

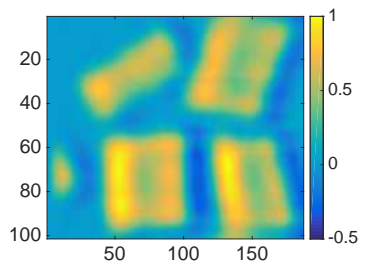

(b) Restored by ZA-LMS

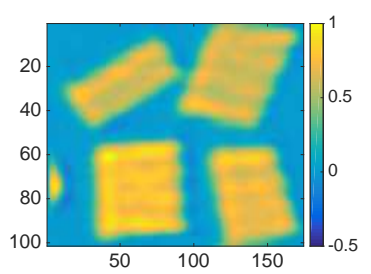

(d) Restored by SBZA-LMS

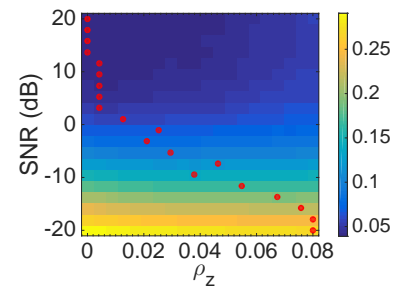

(f) MSE as a function $\rho_{z}$ $(\mu=0.006, Q=15)$
Fig. 2: Influence of different hyperparameters

from each pixel of the hyperspectral image. The imaged object on the background was an integrated circuit including pieces of metal and electronic components. The convolution filter was estimated from data (using a calibration target) to be a Gaussian kernel of size $21 \times 21$. Its full width at half-maximum was 10 pixels. To avoid the storage of matrix $\mathbf{H}_{\ell}$, convolution was performed in the frequency domain. Figure 3 presents the deconvolution result obtained on the whole real hyperspectral image (only 3 slices corresponding to wavelengths $501.1 \mathrm{~nm}$, $672.6 \mathrm{~nm}$ and $844.1 \mathrm{~nm}$ are shown). The coefficients $c_{p}$ were all set to 1 . The original image is shown in Figure 3(a). The image restored with SBZA-LMS $(\mu=0.008, Q=21$, $\left.\rho_{z}=3 \cdot 10^{-4}, \eta_{\lambda}=0.001\right)$ is shown in Figure 3(b). The restored images are of better quality from both denoising and deblurring points of view. The SBZA-LMS result is compared to classical off-line deconvolution algorithms proposed in [14]. Figure 3(c) corresponds to the Tikhonov approach with $\ell_{2}$ spatial and spectral regularizers and Figure 3(d) corresponds to the non-negative version of the Tikhonov approach. The hyperparameters of the non-negative Tikhonov approach were estimated by the minimum distance criterion proposed in [25] and the same values were used for the standard Tikhonov approach. The results of the sequential deconvolution have almost the same resolution compared to both Tikhonov approaches. The noise level on the background is similar to that of the non-negative Tikhonov but the computational burden of the SBZA-LMS remains of the same order with that of the standard Tikhonov and is much lower than that of the nonnegative Tikhonov.
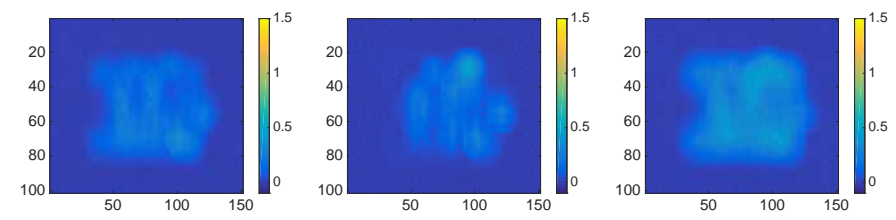

(a) Original hyperspectral image
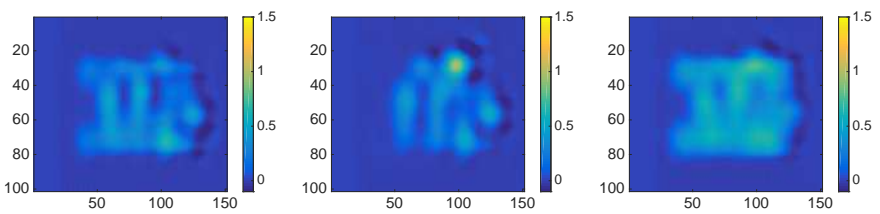

(b) Estimated image (SBZA-LMS)
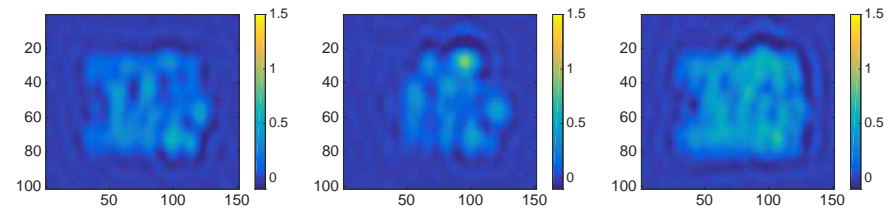

(c) Estimated image (Tikhonov)
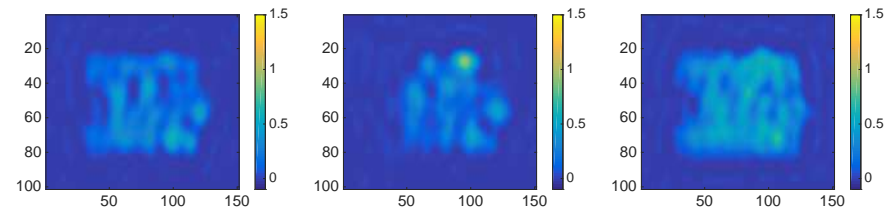

(d) Estimated image (Non-negative Tikhonov)

Fig. 3: Hyperspectral image restoration at 3 wavelengths.

\section{CONCLUSion}

In this work, we addressed the online deconvolution problem of hyperspectral images collected by pushbroom imaging systems. We discussed some issues related to the non-causality of the model. Then, we proposed the SBZA-LMS and the influence of the hyperparameters were assessed. We evaluated the performance of the algorithm by comparing it with the state-of-the-art on real hyperspectral data. The SBZA-LMS improves the data resolution right after each line scanning with a reasonable computational burden. Future works will focus on the derivation of an algorithm to estimate the hyperparameters in an automatic manner. 


\section{REFERENCES}

[1] J. Chen, C. Richard, and P. Honeine, "Nonlinear unmixing of hyperspectral data based on a linear-mixture/nonlinear-fluctuation model," IEEE Transactions on Signal Processing, vol. 61, no. 2, pp. 480-492, 2013.

[2] R. Ammanouil, A. Ferrari, C. Richard, and D. Mary, "Blind and fully constrained unmixing of hyperspectral images," IEEE Transactions on Image Processing, vol. 23, no. 12, pp. 5510-5518, 2014.

[3] R. M. Willett, M. F. Duarte, M. A. Davenport, and R. G. Baraniuk, "Sparsity and structure in hyperspectral imaging: sensing, reconstruction, and target detection," IEEE Signal Processing Magazine, vol. 31, no. 1, pp. 116-126, 2014.

[4] Q. Li, X. He, Y. Wang, H. Liu, D. Xu, and F. Guo, "Review of spectral imaging technology in biomedical engineering: achievements and challenges," Journal of biomedical optics, vol. 18, no. 10, pp. 100 901-100 901, 2013.

[5] Y. R. Chen, R. W. Huffman, B. Park, and M. Nguyen, "Transportable spectrophotometer system for on-line classification of poultry carcasses," Applied Spectroscopy, vol. 50, no. 7, pp. 910-916, 1996.

[6] H. Huang, L. Liu, and M. O. Ngadi, "Recent developments in hyperspectral imaging for assessment of food quality and safety," Sensors, vol. 14, no. 4, pp. 7248-7276, 2014.

[7] C. Cariou and K. Chehdi, "Automatic georeferencing of airborne pushbroom scanner images with missing ancillary data using mutual information," IEEE Transactions on Geoscience and Remote Sensing, vol. 46, no. 5, pp. 1290-1300, 2008.

[8] P. Tatzer, M. Wolf, and T. Panner, "Industrial application for inline material sorting using hyperspectral imaging in the NIR range," RealTime Imaging, vol. 11, no. 2, pp. 99-107, 2005.

[9] Pellenc Selective Technology, "Mistral product," http://www.pellencst.com/products, accessed: 201609-01.

[10] B. R. Hunt and O. Kubler, "Karhunen-Loeve multispectral image restoration, part I: Theory," IEEE Transactions on Acoustics, Speech, and Signal Processing, vol. 32, no. 3, pp. 592-600, 1984.

[11] N. P. Galatsanos, A. K. Katsaggelos, R. T. Chin, and A. D. Hillery, "Digital restoration of multichannel images," IEEE Transactions on Acoustics, Speech, and Signal Processing, vol. 37, no. 3, pp. 415-421, 1989.

[12] _ - "Least squares restoration of multichannel images," IEEE Transactions on Signal Processing, vol. 39, no. 10, pp. 2222-2236, 1991.

[13] J. F. Giovannelli and A. Coulais, "Positive deconvolution for superimposed extended source and point sources," Astronomy \& Astrophysics, vol. 439 , no. 1 , pp. 401-412, 2005.

[14] S. Henrot, C. Soussen, and D. Brie, "Fast positive deconvolution of hyperspectral images," IEEE Transactions on Image Processing, vol. 22, no. 2, pp. 828-833, 2013.

[15] X. Zhao, F. Wang, T. Huang, M. K. Ng, and R. J. Plemmons, "Deblurring and sparse unmixing for hyperspectral images," IEEE Transactions on Geoscience and Remote Sensing, vol. 51, no. 7, pp. 4045-4058, 2013.

[16] J. Jemec, F. Pernuš, B. Likar, and M. Bürmen, "Push-broom hyperspectral image calibration and enhancement by $2 \mathrm{D}$ deconvolution with a variant response function estimate," Optics Express, vol. 22, no. 22, pp. $27655-27668,2014$.

[17] — "Deconvolution-based restoration of SWIR pushbroom imaging spectrometer images," Optics Express, vol. 24, no. 21, pp. 24704$24718,2016$.

[18] B. Widrow and S. D. Stearns, Adaptive signal processing. Englewood Cliffs, NJ, Prentice-Hall, Inc., 1985

[19] Y. Chen, Y. Gu, and A. O. Hero, "Regularized least-mean-square algorithms," arXiv preprint arXiv:1012.5066, 2010.

[20] J. Chen, C. Richard, J.-C. M. Bermudez, and P. Honeine, "Nonnegative least-mean-square algorithm," IEEE Transactions on Signal Processing, vol. 59, no. 11, pp. 5225-5235, 2011.

[21] M. Elad and A. Feuer, "Superresolution restoration of an image sequence: adaptive filtering approach," IEEE Transactions on Image Processing, vol. 8, no. 3, pp. 387-395, 1999.

[22] G. H. Costa and J.-C. M. Bermudez, "Statistical analysis of the LMS algorithm applied to super-resolution image reconstruction," IEEE
Transactions on Signal Processing, vol. 55, no. 5, pp. 2084-2095, May 2007.

[23] Y. Song, E.-H. Djermoune, J. Chen, C. Richard, and D. Brie, "Online deconvolution for pushbroom hyperspectral imaging systems: LMS algorithms and stochastic analysis," CRAN, Research Report, 2017.

[24] S. Zhang and J. Zhang, "Transient analysis of zero attracting NLMS algorithm without gaussian inputs assumption," Signal Processing, vol. 97, pp. 100-109, 2014.

[25] Y. Song, D. Brie, E.-H. Djermoune, and S. Henrot, "Regularization parameter estimation for non-negative hyperspectral image deconvolution," IEEE Transactions on Image Processing, vol. 25, no. 11, pp. 5316-5330, 2016. 